\title{
The Main Injector Beam Position Monitor Front-End Software
}

\author{
Luciano Piccoli, Stephen Foulkes, Margaret Votava and Charles Briegel
}

Fermi National Accelerator Laboratory

P.O. Box 500

Batavia, IL, USA 60510

\begin{abstract}
The front-end software developed for the Main Injector (MI) BPM upgrade is described. The software is responsible for controlling a VME crate, equipped with a Motorola PowerPC board running the VxWorks operating system, a custom made timing board and up to 10 commercial digitizer boards. The complete MI BPM system is composed of 7 independent units, each collecting data from 19 to 38 BPM pickups. The MI BPM system uses several components already employed on the successful upgrade of another Fermilab machine, the Tevatron. The front-end software framework developed for the Tevatron BPM upgrade is the base for building the MI front-end software. The framework is implemented in $\mathrm{C}++$ as a generic component library (GBPM) that provides an event-driven data acquisition environment. Functionality of GBPM is extended to meet MI BPM requirements, such as the ability to handle and manage data from multiple cycles; perform readout of the digitizer boards without disrupting or missing subsequent cycles; transition between closed orbit and turn-by-turn modes within a cycle, using different filter and timing configurations; and allow the definition of new cycles during normal operation.
\end{abstract}

Keywords: Beam Position Monitor.

PACS: 07.77.Ka

\section{INTRODUCTION}

The Main Injector (MI) is a complex particle accelerator used for several experiments at Fermilab. The machine has the ability to accelerate protons and antiprotons from $8 \mathrm{GeV}$ to $150 \mathrm{GeV}$. Protons from the Main Injector are used for anti proton production, as well as neutrino production. The Main Injector is also used for moving antiprotons between the Accumulator, Recycler and Tevatron for collider operations.

The front-end software is one of the components developed for the ongoing MI Beam Position Monitor (BPM) upgrade. The functionality of the software system is based on the recent upgrade to Fermilab's Tevatron [1].

The following section describes the components of the upgraded BPM system; software requirements; existing framework developed as a basis for the Tevatron BPM upgrade; extensions for supporting unique MI abilities and modes of operation; performance measurements and conclusion. 


\section{FRONT-END SYSTEM OVERVIEW}

The upgraded MI BPM system is composed of components located in the tunnel, close to the BPM pickups and in service buildings. In the tunnel, the signals harvested by the pickups are merged by a combiner box (Figure 1, part a) and sent to service buildings via cables of varying sizes (100 ft. to $1200 \mathrm{ft}$.). There are seven service buildings located around the MI ring, each receiving signals from 19 to 38 pickups.

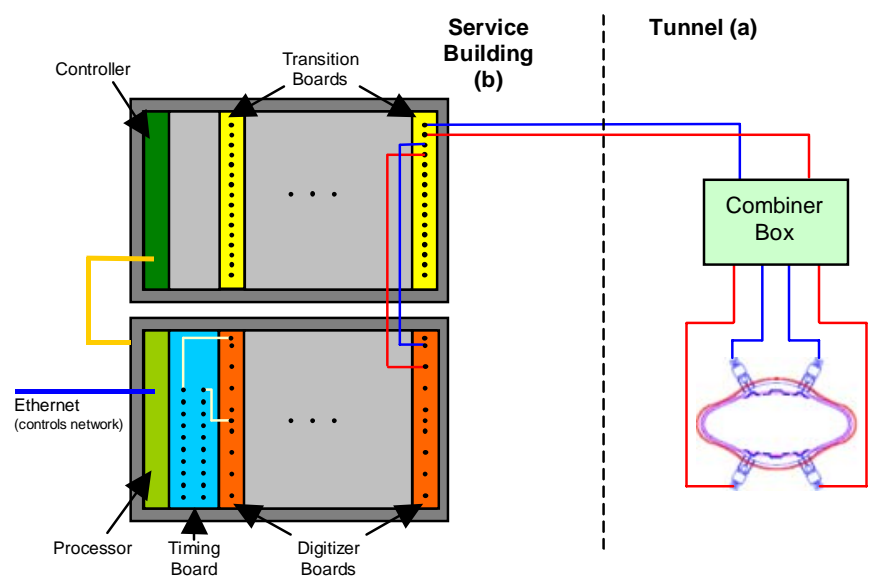

FIGURE 1. Main Injector BPM upgraded hardware components. Signal harvested from the pickup are combined while in the tunnel and sent upstream to the transition boards in the service building. Transition board outputs are connected to digitizer boards controlled by the front-end software.

Each service building has two VME crates (Figure 1, part b). The first crate contains a set of transition boards [2], which receive the signals from the combiner boxes. The crate controller card receives commands from the main crate and configures the transition boards to output either the $2.5 \mathrm{MHz}$ or the $53 \mathrm{MHz}$ signals coming from the combiner boxes.

The main crate contains a processor board, a custom made timing board and commercial digitizer boards. The processor board chosen for the MI BPM upgrade is the Motorola ${ }^{\mathrm{TM}}$ MVME5500-0163 with 512 MB of memory. It runs the VxWorks ${ }^{\circledR}$ Real Time operating system (version 5.5). Due to the potentially high amount of data to be collected this board was chosen because it is possible to install a memory extension of $512 \mathrm{MB}$, totaling $1 \mathrm{~GB}$.

Next to the processor board sits the custom made timing board. It is based on the board developed for the Tevatron BPM upgrade, with minor hardware modifications and additional firmware functionality. The board generates timing signals that control the acquisition of the BPM signals. Timing signals are based on inputs from the accelerator controls clock system, which include signals synchronous to the beam: Main Injector RF clock (RFCLK); the Main Injector beam sync clock (MIBS); the Recycler beam sync clock (RRBS); the Booster extraction signal (BES); and asynchronous signals: Machine Data (MDAT) and the Tevatron event clock (TCLK). The board provides synchronization signals for up to ten digitizer boards. Signals to 
the digitizer boards are propagated through individual cable connections between the boards.

The digitizer boards are commercial boards from Echotek ${ }^{\mathrm{TM}}$ corporation (model ECDR-GC814-FV-2) [3], containing high-speed digitizers for the analog inputs. The board also contains a digital down converter, which produces in-phase (I) and inquadrature (Q) outputs. Each sample is represented by one I/Q value pair. One digitizer has eight input channels, which handle signals from four BPM pickups.

\section{SOFTWARE REQUIREMENTS}

The upgraded BPM system for the Main Injector has requirements that extend the functionality of the original system, while functioning in a similar manner in order to minimize disruptions and avoid redesigning the user interfaces.

Basic data acquisition modes that must be supported by the front-end system are turn-by-turn and closed orbit modes, gathering data from particles bunched at either 53 $\mathrm{MHz}$ or $2.5 \mathrm{MHz}$.

The operating mode of the front-end system is defined by the Main Injector state, also called cycle. A number of injections and extractions may occur during a cycle, and the front-end must to be programmed to arm the desired measurements at the correct time.

On injections and extractions the system is set up for taking turn-by-turn measurements, each of which measures 512 consecutive turns starting at a given bucket. Injections and extractions are armed by accelerator clock signals (BES, MIBS or RRBS). The system is able to collect up to 20 of these measurements, also referred to as flash turn-by-turn, during a single cycle.

Between injections and extractions the system is able to take closed orbit measurements at $500 \mathrm{~Hz}$ and allow beam position and intensity to be calculated. Closed orbit measurements can be stored in separate buffers for later retrieval at the reception of predefined TCLK events.

For every MI state, the front-end software must keep all data collected from the last instance of a state. These are overwritten every time a new instance of the same state is started.

The following section describes some key aspects of the GBPM framework, which is used as basis for the MI BPM front-end software.

\section{THE GENERIC BPM FRAMEWORK}

Most of the basic functionality required for the MI BPM front-end software is implemented by a generic framework (GBPM) developed for the Tevatron BPM upgrade [4]. GBPM defines modules that allow the composition of an event-driven data acquisition. Figure 2 shows how the components interact with each other to form a simple data acquisition system. 


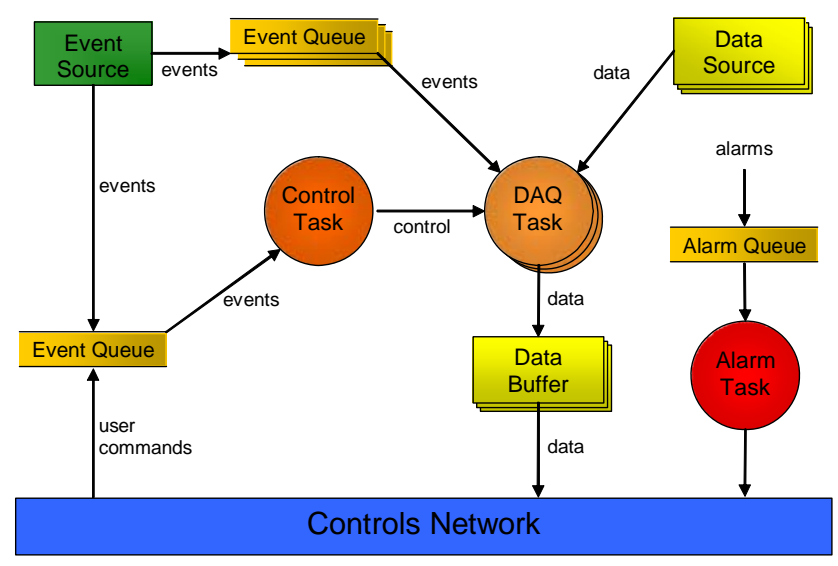

FIGURE 2. Generic Framework developed for the Tevatron BPM upgraded and reused for the MI BPM upgrade project.

Any data acquisition is started by an event, which can be defined, for example, as a timer interrupt or a user command. The event is passed onto one of the active entities in the system: control task, data acquisition task or alarm task. The tasks are constantly waiting for events coming through event queues. The reception of an event triggers the data acquisition and any required processing.

The sequence of data acquisition takes the following form: suppose that an event source (timer interrupt at $1 \mathrm{~Hz}$ ) is programmed to generate events. The event is passed onto any tasks that are listening for events from the generator. One of the listening tasks can start collecting data from a device at the reception the event and re-arm the device after reading it out.

The GBPM framework also abstracts the process of reading out a device and saving the data into memory. Any entity in the system capable of generating data is called a data source and any entity that can store data is a data buffer.

The alarm task is dedicated to receive alarm events for any other part of the system. According to the alarm received the task may forward it to users through the controls network layer.

The controls network layer is the connection between the framework and external applications. The GBPM supports primarily ACNET, however it is possible to extend it to use other control system, such as EPICS.

In order to meet the front-end software requirements, the GBPM functionality was extended. The following section describes the additions.

\section{MIBPM EXTENSION}

The GBPM used as a base for the MI BPM front-end software required the addition of a few capabilities. The constant and fast transition between MI cycles requires the front-end to reconfigure itself to quickly handle distinct data taking configurations. Data from states must be kept in memory until overwritten by another instance of the state and must be available for applications via the controls network. 


\section{Multiple state support}

A Main Injector state is identified by a combination of TCLK and MDAT events. When the front-end detects a state change TCLK the next state number is available from MDAT. This combination of signals defines the next series of data taking commands to be executed.

The list of commands for all states is sent through the controls network to all MI BPM front-ends, making sure all have the same set of instructions, as shown in figure 3.

Commands available to operators are: filter, closed orbit, flash, turn-by-turn and raw. The filter command allows the operator to select the transition module frequency (2.5 $\mathrm{MHz}$ or $53 \mathrm{MHz}$ ), as well as attenuation; closed orbit enables the collection of averaged positions at $500 \mathrm{~Hz}$; flash starts a 512 point turn-by-turn measurement; turnby-turn allows the acquisition of beam position on 2048 consecutive turns; and the raw command disables filtering at the digitizer boards, allowing operators to calibrate the timing for turn-by-turn measurements and diagnostic simple problems like a disconnected input.

All commands have a starting time, specified in ms, that is relative to the state change TCLK signal. It is possible to combine all commands, except the turn-by-turn and raw, which cannot be mixed with closed orbits and flash measurements.

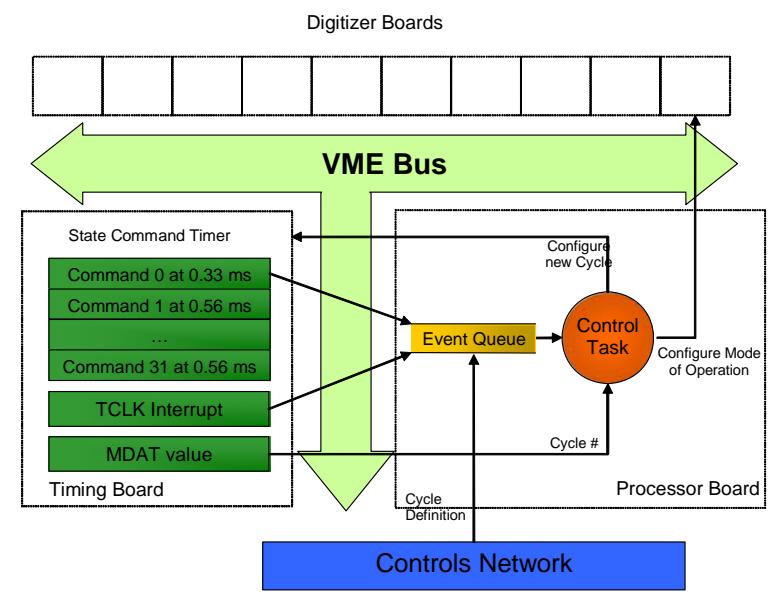

FIGURE 3. Iteration between front-end components for supporting multiple Main Injector states. The list of commands is received from the controls network, which is activated by a TCLK event and identified by an MDAT value. The control task programs the state timer in the timing board and also configures the digitizer for the next measurement.

\section{Buffer organization}

For every defined MI cycle the front-end reserves a set of buffers, which are capable of keeping data for all potential measurements. A buffer set includes closed orbit related buffers: profile (128), display (1), user TCLK (128), flash average (1); and turn-by-turn related buffers: turn-by-turn (2048) and flash turn-by-turn (512). The numbers in parenthesis are the total of samples that each buffer can hold. 
The profile, display and user TCLK buffers pull data from the $500 \mathrm{~Hz}$ closed orbit buffer when a specific TCLK signal is decoded. The profile and display buffers have predefined TCLK trigger events, while the user TCLK buffer has a configurable event. The flash average is a calculated closed orbit measurement based on the first injection flash taken in the cycle.

The system has a pre-allocated buffer pool of flash turn-by-turn buffers, each capable of storing 512 measurements. The variable number of flash turn-by-turn measurements per cycle requires the front-end to use dynamic buffer assignment in order to keep optimum memory usage. A single cycle is allowed have up to 20 flash turn-by-turn buffers used.

\section{Modes of Operation}

The system defines two modes of operation for regular data taking: closed orbit and turn-by-turn modes, and another two modes used for diagnostics: raw and safe modes. All modes of operation can be configured to measure data at $2.5 \mathrm{MHz}$ or $53 \mathrm{MHz}$, depending on the configuration of the transition boards.

\section{Closed Orbit Mode}

Closed orbit measurements are taken by configuring the digitizers to run in an averaged mode (narrow bandwidth mode). The timing system is programmed to generate a trigger for the digitizers at a rate of $500 \mathrm{~Hz}$, represented by the $2 \mathrm{~ms}$ interrupt in figure 4 . After sampling the beam for approximately 40 turns, the software reads out a single $\mathrm{I} / \mathrm{Q}$ value per digitizer channel through the VME backplane and makes it available for plotting through ACNET. If a profile, display of user TCLK is decoded, the closed orbit measurement is also saved into the appropriate buffer.

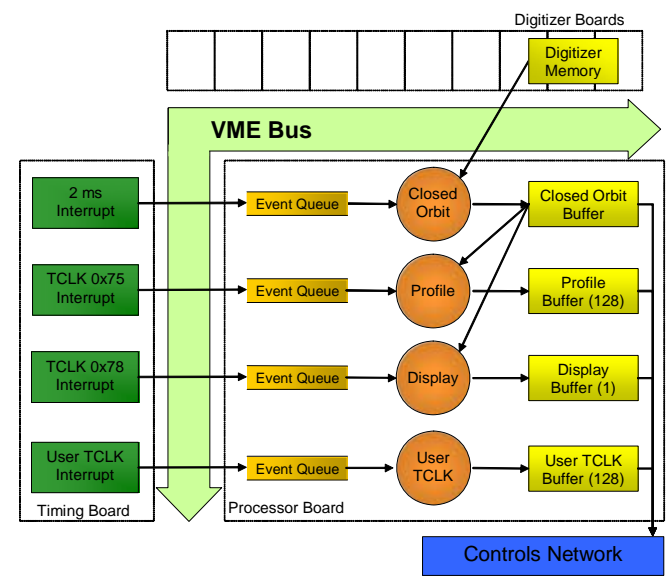

FIGURE 4. Front-end components involved with closed orbit measurements. 
On turn-by-turn mode the digitizers are configured to measure particle positions once every turn (wide bandwidth mode). The timing system is configured to start generating triggers synchronously with the beam. A trigger is issued every turn, to collect data starting at a specific bucket that is configurable by the operator.

Due to timing restrictions and potential high quantity of data, the transfer from the digitizers to the processor board are delayed until the cycle ends, at the reception of an end-of-beam TCLK signal.

After the data transfer is complete, the measurements are routed to the appropriate buffers. For injections and extractions data is copied to the flash turn-by-turn buffers (512 points each), while for user triggered turn-by-turn the data is saved into the turnby-turn buffer (2048 points).

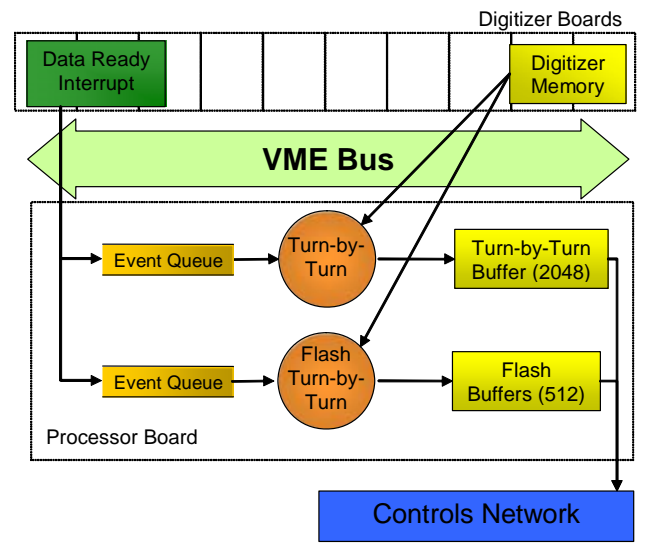

FIGURE 5. Front-end components involved with closed orbit measurements.

\section{Raw Mode}

Raw mode samples the beam at the maximum digitizer frequency, about $75 \mathrm{Mhz}$, with all filtering turned off on the digitizer card. The readout also occurs at the end-ofbeam TCLK and data is saved into a special buffer that is accessible only through a diagnostics application. The raw mode can be used to define the timing parameters for the system and helps to detect faulty connections.

\section{Safe Mode}

The safe mode is designed to be used as an option when the system is not receiving reliable synchronization signals, or when timing has changed. In this mode the digitizers are configured in wide bandwidth mode and $12 \mathrm{~K}$ measurements are taken continuously, instead of one measurement per turn.

The processor uses an algorithm to search for intensity peaks on the data samples. The selected measurements are saved into the first flash turn-by-turn buffer. 


\section{Digitizer Configuration}

Most functionality of the digitizer device driver from the Tevatron BPM upgrade was used for the MI project. However due to timing requirements for MI state data acquisition, the driver functionality was extended.

The digitizer is configured to generate four parallel data streams, with data for closed orbit and turn-by-turn measurements at both $2.5 \mathrm{MHz}$ and $53 \mathrm{MHz}$. The data path for data from a channel pair is depicted in figure 6 .

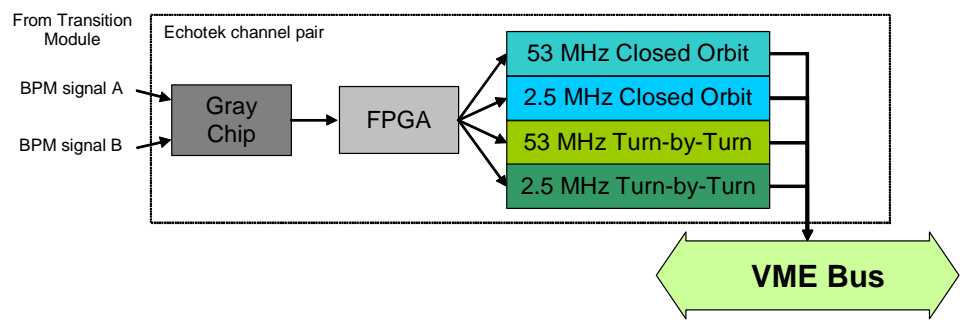

FIGURE 6. In normal data taking the BPM signals pass through the digitizer gray chip and FPGA, which is configured to generate four streams of data, two with closed orbit measurements and two for turn-by-turn measurements.

Closed orbit data (2.5 MHz and $53 \mathrm{MHz}$ ) are always read at $500 \mathrm{~Hz}$, while turn-byturn data is kept within the digitizer memory until the end-of-cycle signal is received. There is enough time between cycles to allow for data to be transferred across the VME backplane into the processor memory.

\section{PERFORMANCE MEASUREMENTS}

The front-end software performance was measured in the test stand setup, which contains a fully configured crate with ten digitizer boards. The timing of the most critical functions was measured and the results are listed below.

The closed orbit measurements are taken at every 2 ms. Most of the time (approximately $1.3 \mathrm{~ms}$ ) is used by the digitizers to collect data. The data transfer time is minimal and does not have any impact on the performance. The remaining $25 \%$ of a closed orbit measurement is used for calculating the position and intensity of the beam based on the I/Q values from the digitizers and also to move data internally to other buffers as needed (e.g. profile and display closed orbit measurements).

During turn-by-turn measurements data is taken for every turn by the digitizers and stored in memory. The transfer to the processor starts only after the current MI cycle finished by the reception of the end-of-beam signal. Between cycles there is a $500 \mathrm{~ms}$ time window that is used to move data from the digitizers into the processor memory. The maximum amount of data to be transferred from the digitizers is $12 \mathrm{~K}$ measurements, and for a fully loaded crate the total transfer time takes $150 \mathrm{~ms}$. The remaining time is also used for position and intensity calculation and for moving data to the correct memory buffers.

During a cycle the front-end is required to switch between closed orbit and turn-byturn mode within $10 \mathrm{~ms}$, which is the time the machine needs to transition between 
bunch structures. The amount of time to transition between different modes was measured at $1.8 \mathrm{~ms}$, allowing plenty of time for any additional processing during a mode change.

\section{CONCLUSION}

The front-end software for the Main Injector BPM upgrade has been described. The system structure uses the framework designed and implemented for the Tevatron BPM upgraded. In order to meet the upgrade requirements the framework had its functionality extended. It also required changes in the digitizer driver and in the timing hardware to support multiple MI cycles.

The system will be commissioned starting in May of 2006 after the machine is back up from a long shutdown. The original BPM system will be used to bring the MI back into operations, except for a fraction of one service building that is under control of the upgraded system.

\section{ACKNOWLEDGMENTS}

This work was supported by Fermi National Accelerator Laboratory, operated by Universities Research Association Inc. under Contract No. DE-AC02-76CH03000 with the United States Department of Energy.

\section{REFERENCES}

1. S. Wolbers et al., Tevatron Beam Position Monitor Upgrade, in Particle Accelerator Conference (PAC05), Knoxville, TN, May 2005.

2. M. Wendt and S. M. Rapizarda and A. Saewert, Analog Signal Pre-Processing for the Fermilab Main Injector BPM upgrade, in BIW06.

3. Echotek Corporation, Echotek ECDR-814x user manual, Huntsville, AL, [Online]. Available: http://www.echotek.com/a2d/ecdr-814 AD/ECDR-814Rev3p0062001.pdf.

4. L. Piccoli, M. Votava and D. Zhang, The New Tevatron Beam Position Monitor Front-End Software, in $14^{\text {th }}$ IEEE-NPSS Real Time Conference, Stockholm, Sweden, June 2005. 